\title{
Ultrasound-Promoted Formation of Isopentenyl Alcohol Dianion: Straightforward Synthesis of Perhydrofuro[2,3-b]furans
}

\author{
Francisco Alonso,* Mamen Rodríguez-Fernández, Daniel Sánchez, Miguel Yus* \\ Departamento de Química Orgánica, Facultad de Ciencias and Instituto de Síntesis Orgánica (ISO), Universidad de Alicante, \\ Apdo. 99, 03080 Alicante, Spain \\ Fax +34(965)903549; E-mail: falonso@ua.es; E-mail: yus@ua.es \\ Received 4 May 2010 \\ Dedicated to the memory of Professor José Manuel Concellón
}

\begin{abstract}
Ultrasound has been found to accelerate the formation of isopentenyl alcohol dianion by metalation with butyllithium in diethyl ether-tetrahydrofuran. The reaction of this dianion with carbonyl compounds followed by intramolecular acetalization under Wacker-type conditions provides a direct route for the synthesis of 2-substituted perhydrofuro[2,3-b]furans.
\end{abstract}

Key words: metalation, ultrasound, polyanions, Wacker-type reaction, cyclization, perhydrofuro[2,3-b]furans

2-Methylprop-2-en-1-ol (methallyl alcohol, 1) and 3-methylbut-3-en-1-ol (isopentenyl alcohol, 2) are inexpensive and commercially available building blocks, which, through a double direct metalation reaction, can be readily transformed into synthetically useful molecules (Figure 1). The formation of dianions $\mathbf{4}$ and $\mathbf{5}$ from $\mathbf{1}$ and 2 was first described in the 1970s using, respectively, Schlosser's base in hexane or butyllithium/TMEDA in hexane. ${ }^{1}$ A modification of the latter procedure was later introduced by Trost et al. by using a diethyl ether-tetrahydrofuran solvent mixture and optimized for the preparation of allylsilanes derived from 1 and $2 .^{2}$ In more recent literature, the direct trimetalation of 2,4-dimethylpenta1,4-dien-3-ol (3) was conducted with sec-butyllithium/ TMEDA in diethyl ether-cyclohexane, with the derived organolithium intermediate $\mathbf{6}$ being trapped with various electrophiles. $^{3}$<smiles>C=C(C)CO</smiles>

1

4<smiles>C=C(CO)CCl</smiles>

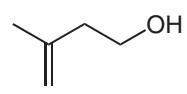

2<smiles>C=C(CCl)CCO</smiles>

5

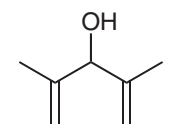

3<smiles>C=C(CCl)C(O)C(=C)CCl</smiles>

6
Figure 1

Perhydrofuro[2,3- $b]$ furans substituted at the 2-position can be found as substructures in clerodane diterpenes, which are especially abundant in Ajuga $a^{4}$ and Scutellaria ${ }^{5}$

SYNTHESIS 2010, No. $x$, pp 0001-0008

Advanced online publication: xx.xx.2010

DOI: 10.1055/s-0030-xxxxx; Art ID: Z11410SS

(C) Georg Thieme Verlag Stuttgart · New York

Imprimatur: species. Some representative examples of this family of natural products are lupulin $\mathrm{C}^{4 \mathrm{a}}(\mathbf{I})$ and scutecolumnin $\mathrm{C}$ (II $^{5 \mathrm{a}}$ (Figure 2). In some cases, a perhydrofuro[2,3- $b$ ]furan-2-one moiety is also present in the clerodanes, arising from the oxidation of the hemiacetal functionality, i.e. III. ${ }^{6}$ These compounds exhibit manifold biological activity, especially as antifeedants of insects. ${ }^{7}$ Model compounds IV and $\mathbf{V}$ are synthetic analogues that were found to display insect antifeedant activity in laboratory bioassays $^{8}$ (Figure 2). In addition, compound $\mathbf{V}(\mathrm{R}=\mathrm{Me})$ is a key intermediate in the synthesis of artificial analogues of mycalamide A. ${ }^{9}$ The reported synthetic routes to compounds of the type $\mathbf{I V}$ and $\mathbf{V}$ are, however, rather long. ${ }^{8-10}$ Alternative approaches to obtain 2-substituted perhydrofuro[2,3-b]furans in a more straightforward manner are, therefore, welcome.
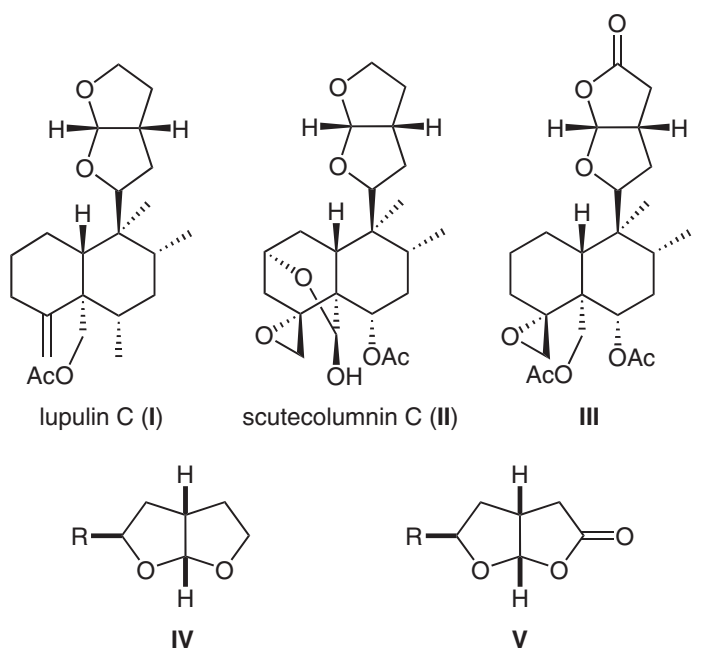

$\mathrm{R}=$ alkyl, cycloalkyl, aryl

scutecolumnin C (II)

III

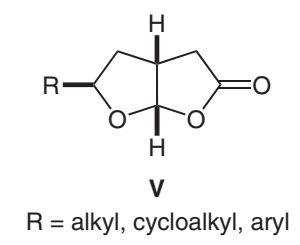

Figure 2

Due to our continued interest in the synthesis of fused bicyclic $^{11}$ and spirocyclic ${ }^{12}$ polyether skeletons, we recently published a highly efficient synthesis of 2,5-substituted perhydrofuro[2,3- $b]$ furans. The strategy was based on the arene-catalyzed lithiation of allylic chlorinated substrates and subsequent reaction with carbonyl compounds, followed by intramolecular acetalization of the resulting 3-methylene-1,5-diols under Wacker-type reaction conditions. ${ }^{13}$ The latter step represents the first palla- 


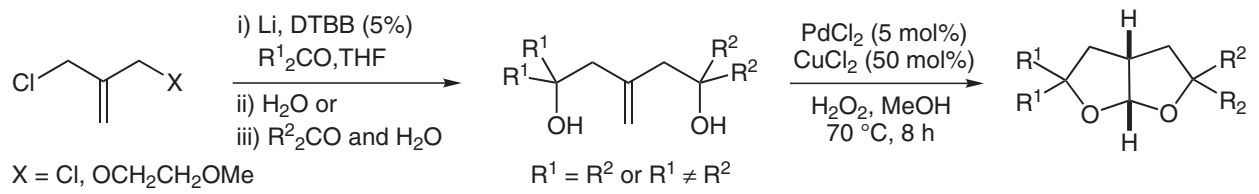

Scheme 1 Synthesis of 2,5-substituted perhydrofuro[2,3-b]furans through arene-catalyzed lithiation and Wacker-type reactions

dium-catalyzed intramolecular acetalization of a dihydroxy-substituted geminal alkene (Scheme 1).

We wish to present herein a new and direct route for the synthesis of 2-substituted perhydrofuro[2,3-b]furans involving the generation of isopentenyl alcohol dianion under ultrasound irradiation and intramolecular cyclization under Wacker-type reaction conditions. A protocol for the direct oxidation of the perhydrofuro[2,3-b]furan moiety to the corresponding lactone has been also studied.

Initial attempts to obtain the precursor diols following a similar strategy to the aforementioned route was rather long and, therefore, not very efficient (Scheme 2). The whole process involved protection of isopentenyl alcohol, allylic chlorination, ${ }^{14}$ arene-catalyzed lithiation ${ }^{15}$ in the presence of a carbonyl compound, and deprotection. Instead, we decided to use isopentenyl alcohol (2) as the direct source of dianion $\mathbf{5}$ and study its reactivity with carbonyl compounds. The reaction of methallyl alcohol dianion 4 with ketones was described by Carlson, ${ }^{1 \mathrm{~b}}$ although the yields of the corresponding diols were modest $(15-40 \%)$. The reactivity of trianion $\mathbf{6}$ was not tested with carbonyl compounds ${ }^{3}$ whereas that of dianion 5 was mainly reported with chlorosilanes ${ }^{2}$ and ally ${ }^{1 \mathrm{a}}$ and alkyl halides. ${ }^{16}$ To the best of our knowledge, there is only one example in the literature of the reaction of dianion $\mathbf{5}$ with carbonyl compounds (two aldehydes), as the key step in the synthesis of vitamin A and methyl $(2 E, 4 E)-3,7,11$-trimethyldodeca-2,4-dienoate. ${ }^{17}$ Therefore, it would be of interest to study the generation and reactivity of dianion 5 with carbonyl compounds in more detail.

We first tested the reaction conditions as reported by Trost et al. for the synthesis of allylsilanes $\mathbf{7}$ and $\mathbf{8}$ from $\mathbf{1}$ and $\mathbf{2}$; they were both obtained in 52\% isolated (Scheme 3 ). ${ }^{2}$ The only objection to this methodology is the long reaction time required for the generation of the corresponding dianions $\mathbf{4}$ and $\mathbf{5}$, respectively. We observed, however, that the reaction times were notably shortened under ultrasound irradiation ${ }^{18}$ whilst maintaining the product yields (Scheme 4).

Based on this methodology, a variety of reaction conditions were screened in order to optimize both the dianion

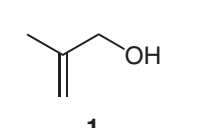<smiles>C=C(C)CCO</smiles>

2
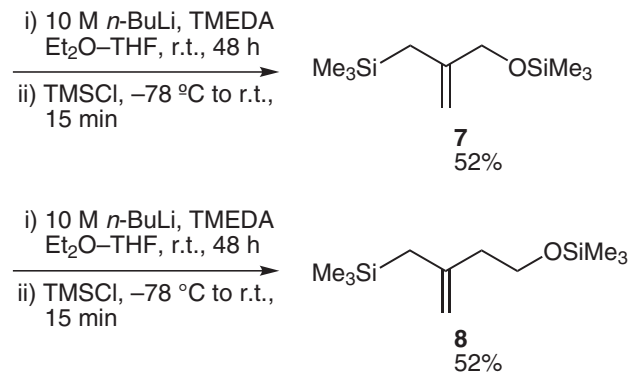

Scheme 3 Synthesis of allylsilanes $\mathbf{7}$ and $\mathbf{8}$ through conventional metalation conditions

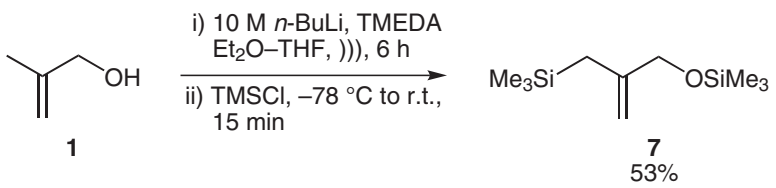

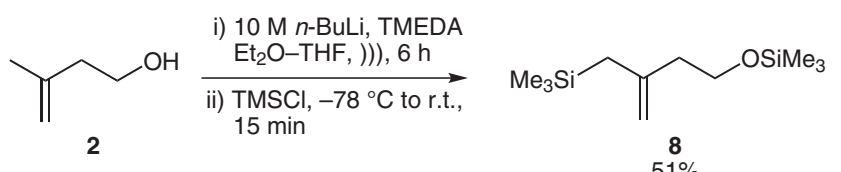

Scheme 4 Synthesis of allylsilanes 7 and $\mathbf{8}$ through ultrasound-promoted dianion formation

formation of $\mathbf{2}$ and its reactivity toward carbonyl compounds, using pentan-3-one as the model electrophile (Table 1). Within the different experiments performed with $10 \mathrm{M}$ butyllithium in diethyl ether-tetrahydrofuran and TMEDA at room temperature (entries 1-5), the best results were obtained using 5.2 equivalents of TMEDA and 3.0 equivalents of pentan-3-one either (entry 4). The slow addition of the electrophile at $-78{ }^{\circ} \mathrm{C}$ (entry 5), however, minimized the formation of byproducts when carbonyl compounds other than pentan-3-one were used, especially, aldehydes. Different reaction conditions reported in the literature, some of which for polyanion formation and reaction with some other electrophiles, gave poorer yields and/or complex mixtures. Such is the case for butyllithium in diethyl ether-TMEDA (entry 6), ${ }^{16}$ toluene (entry 7), ${ }^{19}$ or hexane-TMEDA (entry 8$) ;{ }^{17}$ sec-Butyllithium in diethyl ether-cyclohexane-TMEDA (entry

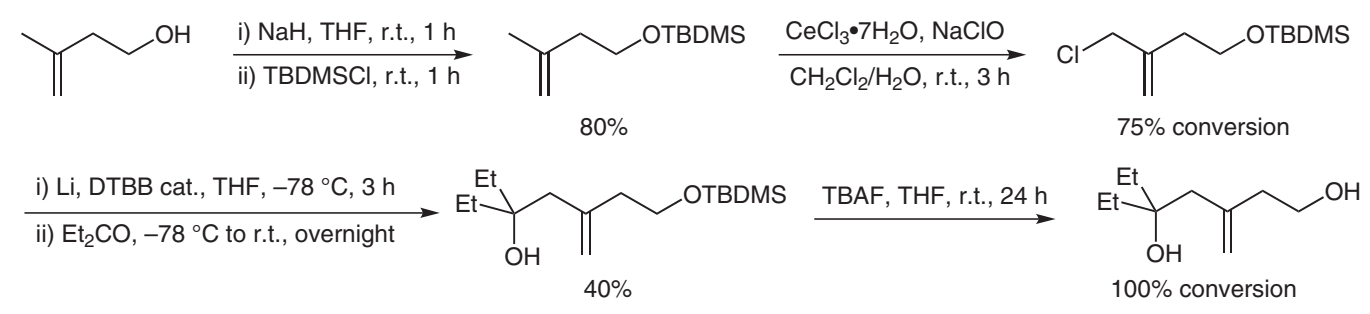

Scheme 2 Preliminary synthesis of a 3-methylene-1,5-diol precursor of a 2-substituted perhydrofuro[2,3-b]furan 
Table 1 Optimization of the Double Metalation of 2 and Reaction with Pentan-3-one ${ }^{\mathrm{a}}$

\begin{tabular}{|c|c|c|c|c|c|}
\hline 2 & & $9 a$ & & & \\
\hline Entry & Base (equiv) & Solvent, conditions & Additive (equiv) & Equiv of $\mathrm{Et}_{2} \mathrm{CO}$ & Yield $^{\mathrm{b}}(\%)$ \\
\hline 1 & $10 \mathrm{M} \mathrm{BuLi}(3.0)$ & $\mathrm{Et}_{2} \mathrm{O}-\mathrm{THF}(3: 2), \mathrm{MW}$ & TMEDA (2.6) & 1.0 & 35 \\
\hline 2 & $10 \mathrm{M} \mathrm{BuLi}(3.0)$ & $\mathrm{Et}_{2} \mathrm{O}-\mathrm{THF}(3: 2), \mathrm{MW}$ & TMEDA (2.6) & 2.0 & 44 \\
\hline 3 & $10 \mathrm{M} \mathrm{BuLi}(3.0)$ & $\mathrm{Et}_{2} \mathrm{O}-\mathrm{THF}(3: 2), \mathrm{MW}$ & TMEDA (5.2) & 2.0 & 48 \\
\hline 4 & $10 \mathrm{M} \mathrm{BuLi}(3.0)$ & $\mathrm{Et}_{2} \mathrm{O}-\mathrm{THF}(3: 2), \mathrm{MW}$ & TMEDA (5.2) & 3.0 & 58 \\
\hline 5 & $10 \mathrm{M} \mathrm{BuLi}(3.0)$ & $\mathrm{Et}_{2} \mathrm{O}-\mathrm{THF}(3: 2), \mathrm{MW}$ & TMEDA (5.2) & $3.0^{\mathrm{c}}$ & 58 \\
\hline $6^{16}$ & $2 \mathrm{M}$ BuLi (2.0) & $\mathrm{Et}_{2} \mathrm{O}$ & TMEDA (2.6) & 0.5 & complex mixture \\
\hline $7^{19}$ & $10 \mathrm{M} \mathrm{BuLi}(3.0)$ & toluene, MW & - & 1.0 & 24 \\
\hline $8^{17}$ & 2.5 M BuLi (2.0) & hexane & TMEDA (2.0) & 1.0 & 37 \\
\hline $9^{3}$ & 1.4 M s-BuLi (4.7) & $\mathrm{Et}_{2} \mathrm{O}-$ cyclohexane $(3: 2)$ & TMEDA (5.3) & 3.0 & complex mixture \\
\hline 10 & 1.7 M t -BuLi (3.0) & $\mathrm{Et}_{2} \mathrm{O}-\mathrm{THF}(3: 2), \mathrm{MW}$ & TMEDA (5.2) & 3.0 & complex mixture \\
\hline $11^{20}$ & $\mathrm{Li}\left(\mathrm{CH}_{2}\right)_{4} \mathrm{OLi}^{\mathrm{d}}(3.5)$ & THF, MW & - & 1.0 & 23 \\
\hline
\end{tabular}

${ }^{a}$ Dianion formation at r.t. for $6 \mathrm{~h}\left(12 \mathrm{~h}\right.$ in entry $8,4 \mathrm{~h}$ in entry 9), followed by reaction with pentan-3-one at $-78^{\circ} \mathrm{C}$ to r.t. overnight.

${ }^{\mathrm{b}}$ Isolated yield after column chromatography.

${ }^{\mathrm{c}}$ Slow addition of the ketone $\left(-78^{\circ} \mathrm{C}\right.$ for $\left.3 \mathrm{~h}\right)$, overnight at $-78^{\circ} \mathrm{C}$ to r.t.

${ }^{\mathrm{d}}$ Generated from THF and Li-DTBB. ${ }^{20}$

9), ${ }^{3}$ tert-butyllithium in diethyl ether-tetrahydrofuranTMEDA (entry 10) or the dianion resulting from the reductive cleavage of tetrahydrofuran with lithium $4,4^{\prime}$-ditert-butylbiphenyl (Li-DTBB) (entry 11). ${ }^{20}$

The optimized reaction conditions were extended to other carbonyl compounds, including three different ketones and aldehydes bearing alkyl, cycloalkyl, and aryl substituents (Scheme 5, Table 2). Within the ketones, pentan-3one gave the best result (entry 1), with the methylene diols 9b and 9c derived from cyclohexanone and benzophenone, respectively, being obtained in modest yields (entries 2 and 3). A similar performance was observed for valeraldehyde, cyclohexanecarbaldehyde, and benzaldehyde, which gave 9d-f in 32-66\% yield (entries 3-6). Notwithstanding the low yields, the fact that the reaction could be carried on a 10-mmol scale allowed the methylene diols 9 to be obtained in substantial and practical amounts.

Diols 9 were subjected to intramolecular acetalization under Wacker-type reaction conditions as previously described by us (Scheme 6, Table 2). ${ }^{13}$ The diols derived

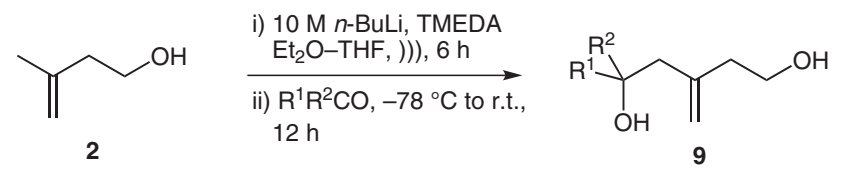

$\mathrm{R}^{1} \mathrm{R}^{2} \mathrm{CO}=\mathrm{Et}_{2} \mathrm{CO},\left(\mathrm{CH}_{2}\right)_{5} \mathrm{CO}, \mathrm{Ph}_{2} \mathrm{CO}, \mathrm{Me}\left(\mathrm{CH}_{2}\right)_{3} \mathrm{CHO}, c-\mathrm{C}_{6} \mathrm{H}_{11} \mathrm{CHO}, \mathrm{PhCHO}$

Scheme 5 Reaction of isopentenyl alcohol dianion with various carbonyl compounds from pentan-3-one 9a and cyclohexanone 9b cyclized nicely to afford the corresponding perhydrofuro[2,3$b$ ]furans 10a and 10b in high yields (entries 1 and 2). In contrast, diol 8c derived from benzophenone gave the product 10c in modest yield (entry 3 ). Acetalization of diols derived from aldehydes 9 d-f proceeded stereoselectively in modest-to-moderate isolated yields (entries 4-6). A maximum 93:7 diastereromeric ratio was reached in the acetalization of the diol derived from cyclohexanecarbaldehyde 9e (entry 5). The major $\left(2 R^{*}, 3 \mathrm{a} S^{*}, 6 \mathrm{a} R^{*}\right)$ relative configuration observed is in agreement with that previously reported by us for 2,5-substituted perhydrofuro[2,3$b$ ] furans ${ }^{13}$ and was confirmed by NOE experiments conducted on both diastereomers of compound $\mathbf{1 0 f}$ (Figure 3). A small NOE was observed for $\mathrm{H} 2$ and $\mathrm{H} 3 \mathrm{a}$ in both diastereomers, whereas NOE between $\mathrm{H} 2$ and $\mathrm{H} 5$ was manifested only in the major diastereomer. PM3 ${ }^{21}$ geometry optimization revealed the closer location of $\mathrm{H} 2$ and $\mathrm{H} 5$ in $\left(2 R^{*}, 3 \mathrm{a} S^{*}, 6 \mathrm{a} R^{*}\right)-\mathbf{1 0 f}$ compared with that in $\left(2 S^{*}, 3 \mathrm{a} S^{*}, 6 \mathrm{a} R^{*}\right)$-10f (Figure 3$)$. It is noteworthy that quantitative conversion of the starting methylene diols was recorded in all cases. An important loss of mass, however, was observed for compounds $\mathbf{1 0 c}, \mathbf{d}$ during their purification by column chromatography, probably due to partial decomposition. The general longer reaction times required for the formation of 2-substituted or 2,2-disubstituted compounds $\mathbf{1 0}(24 \mathrm{~h})$ in comparison with the 2,5disubstituted or 2,2,5,5-tetrasubstituted counterparts (8 h) ${ }^{13}$ could be attributed somewhat to the gem-dialkyl effect. $^{22}$ 
Table 2 Synthesis of Methylene-Substituted Diols 9 and Perhydrofuro[2,3- $b$ ]furans 10

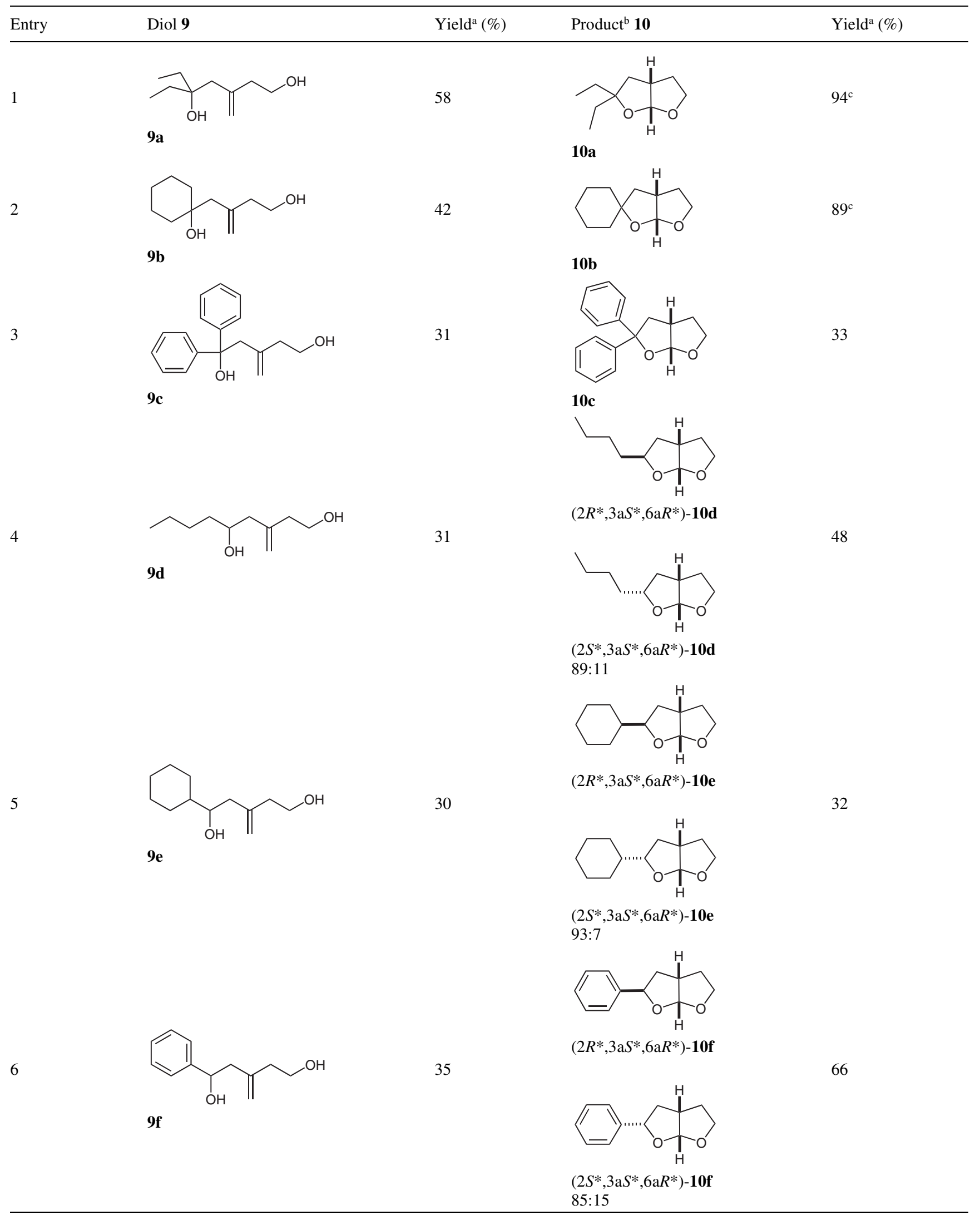

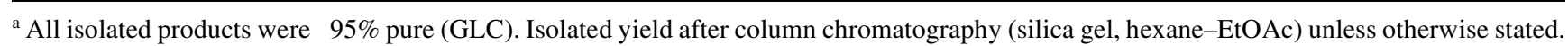
${ }^{b}$ Diastereomeric ratio determined by ${ }^{1} \mathrm{H}$ NMR.

${ }^{\mathrm{c}}$ Reaction crude yield. 


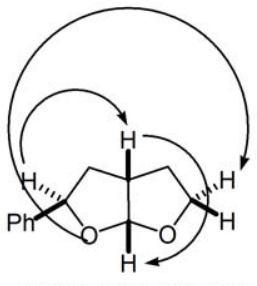

$\left(2 R^{\star}, 3 a S^{*}, 6 a R^{\star}\right)-10 f$
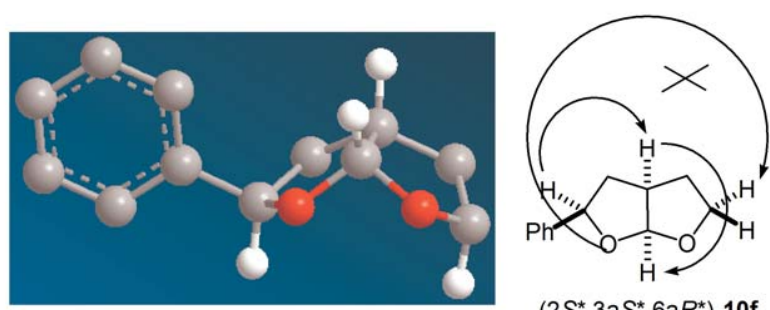

$\left(2 S^{*}, 3 a S^{*}, 6 a R^{\star}\right)-10 f$

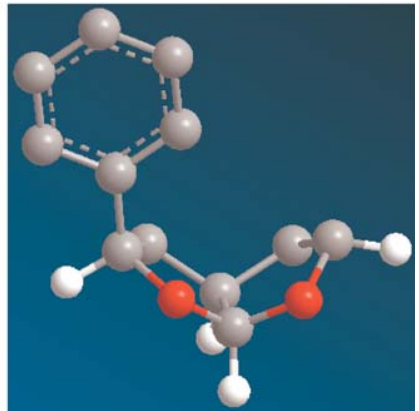

Figure 3 NOE experiments and optimized geometry models for the diastereomeric perhydrofuro[2,3- $b$ ]furans 10f; numbers on the arrows refer to interatomic distances in $\AA$

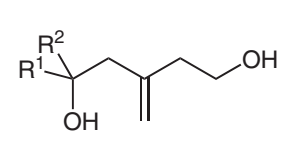

9

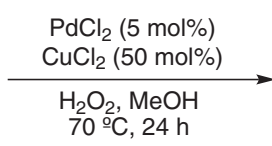

$70 \stackrel{\circ}{\circ}, 24 \mathrm{~h}$

10

Scheme 6 Cyclization of methylene diols 9 to perhydrofuro[2,3$b$ ]furans 10 under Wacker-type conditions

In spite of the fact that the overall yields for compounds $\mathbf{1 0}$ are not as optimum as desired, the methodology presented herein is the most direct route reported so far to this type of compounds. As an example, the synthesis of perhydrofuro[2,3-b]furan 10e described by de Groot et al. ${ }^{8 b}$ involved the noncommercial 4,4-diethoxybutanenitrile and 2-cyclohexyloxirane starting materials in a nine-step sequence. In contrast, only two steps, from commercially available isopentenyl alcohol and cyclohexanecarbaldehyde, were involved in our methodology, which, in addition, resulted in a higher product stereoselectivity (Scheme 7).

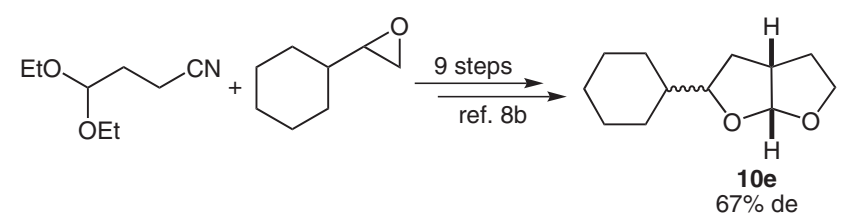<smiles>C=C(C)CCOC1CCC(C=O)CC1</smiles>

Scheme 7 Comparative synthesis of perhydrofuro[2,3-b]furan 10e according to the literature approach ${ }^{8 b}$ and that described herein

Finally, we studied the possibility to access the perhydrofuro[2,3- $b]$ furan-2-one moiety by direct oxidation of the perhydrofuro[2,3- $b]$ furan core of 10a. Among the different conditions tested, the oxidation with catalytic ruthenium(IV) oxide and stoichiometric sodium periodate in the dichloromethane-water-acetonitrile solvent system gave the best outcome of lactone 11a (Scheme 8). ${ }^{22}$

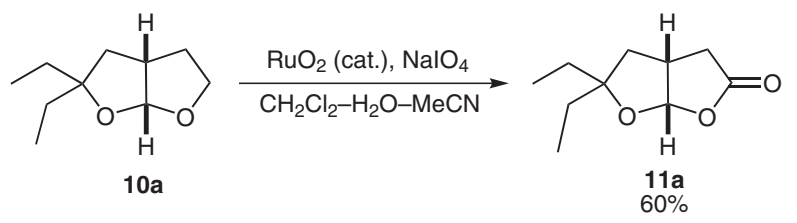

Scheme 8 Oxidation of perhydrofuro[2,3-b]furan 10a to lactone 11a

In conclusion, we have developed a new synthesis of 2substituted perhydrofuro[2,3- $b]$ furans based on the ultrasound-promoted generation of the dianion of isopentenyl alcohol and reaction with carbonyl compounds, followed by palladium-catalyzed intramolecular acetalization under Wacker-type reaction conditions. The methodology has been applied both to ketones and aldehydes, with the perhydrofuro[2,3-b]furans arising from the latter being obtained stereoselectively. Although the overall yields are modest, this approach represents the most direct route to this kind of compounds. Moreover, their transformation into the corresponding lactones can be easily accomplished by ruthenium-catalyzed oxidation. Further studies regarding the reactivity of these compounds are underway.

Melting points were obtained with a Reichert Thermovar apparatus. IR analysis was performed with a FT-IR Nicolet Impact 400D spectrophotometer. NMR spectra were recorded on 300 and 400 spectrometers [300 and $400 \mathrm{MHz}\left({ }^{1} \mathrm{H}\right)$ and 75 and $100 \mathrm{MHz}\left({ }^{13} \mathrm{C}\right)$ ] using $\mathrm{CDCl}_{3}$ as solvent and TMS as internal standard. Mass spectra (EI) were obtained at $70 \mathrm{eV}$ on an Agilent 5973 spectrometer. HRMS analyses were carried out on a Finnigan MAT95S spectrometer. The purity of volatile compounds and the chromatographic analyses (GLC) were determined with a Hewlett Packard HP-6890 instrument equipped with a flame ionization detector and a $30 \mathrm{~m}$ capillary column (0.32 mm diameter, $0.25 \mu \mathrm{m}$ film thickness), using $\mathrm{N}_{2}$ (2 $\mathrm{mL} / \mathrm{min})$ as carrier gas, $T_{\text {injector }}=275^{\circ} \mathrm{C}, T_{\text {column }}=60^{\circ} \mathrm{C}(3 \mathrm{~min})$ and $60-270{ }^{\circ} \mathrm{C}\left(15^{\circ} \mathrm{C} / \mathrm{min}\right)$. Sonication was carried out on a JP Selecta Ultrasons apparatus $(6 \mathrm{~L}, 150 \mathrm{~W}, 40 \mathrm{kHz})$. Flash column chromatography was performed using silica gel $60(40-60 \mu)$. Methallyl alcohol (Aldrich), isopentenyl alcohol (Aldrich), $10 \mathrm{M} \mathrm{BuLi}$ (Aldrich), TMEDA (Alfa Aesar), anhyd $\mathrm{Et}_{2} \mathrm{O}$ (Fluka, 99.8\%, $\mathrm{H}_{2} \mathrm{O}$ $\leq 0.005 \%$ ), the carbonyl compounds (Aldrich, Alfa Aesar), $\mathrm{PdCl}_{2}$ (Merck), $\mathrm{CuCl}_{2}$ (Aldrich), 35\% $\mathrm{H}_{2} \mathrm{O}_{2}$ (Acros), $\mathrm{MeOH}$ (Panreac), $\mathrm{RuO}_{2} \cdot \mathrm{xH}_{2} \mathrm{O}$ (Aldrich), $\mathrm{NaIO}_{4}$ (Riedel de Häen), $\mathrm{CH}_{2} \mathrm{Cl}_{2}$ (Panreac), and $\mathrm{MeCN}$ (Panreac) are commercially available. THF was dried in a Sharlab PS-400-3MD solvent purification system using an alumina column. 


\section{Synthesis of Allylsilanes 7 and 8 and Methylene-1,5-diols 9; General Procedure}

Methallyl alcohol (1, $0.84 \mathrm{~mL}, 10 \mathrm{mmol})$ or 3-methylbut-3-enol (2, $1 \mathrm{~mL}, 10 \mathrm{mmol}$ ) was added dropwise to a soln of $10 \mathrm{M} n$-BuLi (3 $\mathrm{mL}, 30 \mathrm{mmol})$ in anhyd $\mathrm{Et}_{2} \mathrm{O}(12 \mathrm{~mL})$ and anhyd TMEDA $(8 \mathrm{~mL})$ at $0{ }^{\circ} \mathrm{C}$ under an inert atmosphere. Then, anhyd THF $(8 \mathrm{~mL})$ was added to the mixture and it was stirred at $0{ }^{\circ} \mathrm{C}$ for a few min. The flask content was sonicated for $6 \mathrm{~h}$ with formation of a deep red precipitate attributed to the dianion of $\mathbf{2}$. Next, the corresponding electrophile $(30 \mathrm{mmol})$ in anhyd $\mathrm{Et}_{2} \mathrm{O}(5 \mathrm{~mL})$ was slowly added at -78 ${ }^{\circ} \mathrm{C}$ over $3 \mathrm{~h}$ and the mixture was stirred for an additionally $12 \mathrm{~h}$ and the temperature was allowed to reach r.t. The reaction was quenched with $\mathrm{H}_{2} \mathrm{O}(10 \mathrm{~mL})$, acidified with $3 \mathrm{M} \mathrm{HCl}$, and extracted with $\mathrm{Et}_{2} \mathrm{O}(3 \times 40 \mathrm{~mL})$. The resulting combined organic phases were sequentially washed with sat. $\mathrm{CuSO}_{4}$ soln $(2 \times 10 \mathrm{~mL}$, in order to quench the TMEDA) and $\mathrm{H}_{2} \mathrm{O}(2 \times 10 \mathrm{~mL})$. The new organic phase was dried (anhyd $\mathrm{MgSO}_{4}$ ) and the solvent was evaporated (20 mbar) to give a crude product that was purified by column chromatography (silica gel, hexane-EtOAc). The allylsilanes $\mathbf{7}$ and $\mathbf{8}$ were characterized by comparison of their physical and spectroscopic data with those reported in the literature. ${ }^{2}$ New compounds 9 are as given below.

\section{5-Ethyl-3-methyleneheptane-1,5-diol (9a)}

Colorless oil; GLC: $t_{\mathrm{R}}=10.28 \mathrm{~min} ; R_{f}=0.33$ (hexane-EtOAc, $1: 1$ ). IR (film): $3362(\mathrm{OH}), 3073,1638 \mathrm{~cm}^{-1}(\mathrm{CH}=\mathrm{C})$.

${ }^{1} \mathrm{H}$ NMR (300 MHz, $\left.\mathrm{CDCl}_{3}\right): \delta=0.87\left(\mathrm{t}, J=7.6 \mathrm{~Hz}, 6 \mathrm{H}, 2 \mathrm{CH}_{3}\right.$ ), $1.50\left(\mathrm{q}, J=7.6 \mathrm{~Hz}, 4 \mathrm{H}, 2 \mathrm{CH}_{2} \mathrm{CH}_{3}\right), 2.23($ br s, $2 \mathrm{H}, 2 \mathrm{OH}), 2.43(\mathrm{t}$, $\left.J=5.9 \mathrm{~Hz}, 2 \mathrm{H}, \mathrm{CH}_{2} \mathrm{CH}_{2} \mathrm{OH}\right), 3.75(\mathrm{t}, J=5.9 \mathrm{~Hz}, 2 \mathrm{H}$, $\left.\mathrm{CH}_{2} \mathrm{CH}_{2} \mathrm{OH}\right), 4.91,5.00\left(2 \mathrm{~s}, 2 \mathrm{H}, \mathrm{H}_{2} \mathrm{C}=\mathrm{C}\right)$.

${ }^{13} \mathrm{C}$ NMR $\left(75 \mathrm{MHz}, \mathrm{CDCl}_{3}\right): \delta=8.0\left(2 \mathrm{CH}_{3}\right), 30.9\left(2 \mathrm{CH}_{2} \mathrm{CH}_{3}\right)$, $40.4\left(\mathrm{CH}_{2} \mathrm{CH}_{2} \mathrm{OH}\right), 44.2\left(\mathrm{CH}_{2} \mathrm{COH}\right), 61.2\left(\mathrm{CH}_{2} \mathrm{OH}\right), 75.0(\mathrm{COH})$, $116.0\left(\mathrm{H}_{2} \mathrm{C}=\mathrm{C}\right), 143.9\left(\mathrm{C}=\mathrm{CH}_{2}\right)$.

MS (EI): $m / z(\%)=154\left[\mathrm{M}^{+}-\mathrm{H}_{2} \mathrm{O}\right](<1), 87$ (100), 69 (26), 57 (60). HRMS: $m / z\left[\mathrm{M}^{+}\right]$calcd for $\mathrm{C}_{10} \mathrm{H}_{20} \mathrm{O}_{2}: 172.1463 ; m / z\left[\mathrm{M}^{+}-\mathrm{H}_{2} \mathrm{O}\right]$ calcd for $\mathrm{C}_{10} \mathrm{H}_{18} \mathrm{O}$ : 154.1358 ; found: 154.1376 .

\section{1-(4-Hydroxy-2-methylenebutyl)cyclohexanol (9b)}

White solid; $\mathrm{mp} 55{ }^{\circ} \mathrm{C}$; GLC: $t_{\mathrm{R}}=11.90 \mathrm{~min} ; R_{f}=0.44$ (hexaneEtOAc, 1:1).

IR (KBr): $3415(\mathrm{OH}), 3076,1638 \mathrm{~cm}^{-1}(\mathrm{CH}=\mathrm{C})$.

${ }^{1} \mathrm{H}$ NMR $\left(300 \mathrm{MHz}, \mathrm{CDCl}_{3}\right): \delta=1.20-1.70\left[\mathrm{~m}, 10 \mathrm{H},\left(\mathrm{CH}_{2}\right)_{5}\right], 2.24$ (s, $\left.2 \mathrm{H}, \mathrm{CCH}_{2} \mathrm{COH}\right), 2.42\left(\mathrm{t}, J=6.1 \mathrm{~Hz}, 2 \mathrm{H}, \mathrm{CH}_{2} \mathrm{CH}_{2} \mathrm{OH}\right.$ ), 2.51 (br $\mathrm{s}, 2 \mathrm{H}, 2 \mathrm{OH}), 3.75\left(\mathrm{t}, J=6.1 \mathrm{~Hz}, 2 \mathrm{H}, \mathrm{CH}_{2} \mathrm{CH}_{2} \mathrm{OH}\right), 4.91,5.00$ (2 $\left.\mathrm{s}, 2 \mathrm{H}, \mathrm{H}_{2} \mathrm{C}=\mathrm{C}\right)$.

${ }^{13} \mathrm{C}$ NMR $\left(75 \mathrm{MHz}, \mathrm{CDCl}_{3}\right): \delta=22.2\left(2 \mathrm{CH}_{2} \mathrm{CH}_{2} \mathrm{COH}\right), 25.6$ $\left(\mathrm{CH}_{2} \mathrm{CH}_{2} \mathrm{CH}_{2} \mathrm{COH}\right), 37.8\left(2 \mathrm{CH}_{2} \mathrm{COH}\right), 40.5\left(\mathrm{CH}_{2} \mathrm{CH}_{2} \mathrm{OH}\right), 47.7$ $\left(\mathrm{CH}_{2} \mathrm{COH}\right), 61.08\left(\mathrm{CH}_{2} \mathrm{OH}\right), 71.5(\mathrm{COH}), 115.9\left(\mathrm{H}_{2} \mathrm{C}=\mathrm{C}\right), 143.4$ $\left(\mathrm{C}=\mathrm{CH}_{2}\right)$.

MS (EI): $m / z(\%)=166\left[\mathrm{M}^{+}-\mathrm{H}_{2} \mathrm{O}\right](2), 100(10), 99$ (100), 81 (59), 79 (11), 55 (18).

HRMS: $m / z\left[\mathrm{M}^{+}\right]$calcd for $\mathrm{C}_{11} \mathrm{H}_{20} \mathrm{O}_{2}: 184.1463 ; \mathrm{m} / z\left[\mathrm{M}^{+}-\mathrm{H}_{2} \mathrm{O}\right]$ calcd for $\mathrm{C}_{11} \mathrm{H}_{18} \mathrm{O}$ : 166.1358 ; found: 166.1352 .

\section{3-Methylene-1,1-diphenylpentane-1,5-diol (9c)}

Colorless oil; GLC: $t_{\mathrm{R}}=16.70 \mathrm{~min} ; R_{f}=0.61$ (hexane-EtOAc, $1: 1)$.

IR (film): $3382(\mathrm{OH}), 3059,3025,1644,1493 \mathrm{~cm}^{-1}(\mathrm{CH}=\mathrm{C})$.

${ }^{1} \mathrm{H}$ NMR $\left(400 \mathrm{MHz}, \mathrm{CDCl}_{3}\right): \delta=1.94(\mathrm{t}, J=6.1 \mathrm{~Hz}, 2 \mathrm{H}$, $\left.\mathrm{CH}_{2} \mathrm{CH}_{2} \mathrm{OH}\right), 3.13\left(\mathrm{~s}, 2 \mathrm{H}, \mathrm{CH}_{2} \mathrm{COH}\right), 3.78(\mathrm{t}, J=6.1 \mathrm{~Hz}, 2 \mathrm{H}$, $\left.\mathrm{CH}_{2} \mathrm{OH}\right), 4.82,4.96\left(2 \mathrm{~s}, 2 \mathrm{H}, \mathrm{H}_{2} \mathrm{C}=\mathrm{C}\right), 7.15-7.55(\mathrm{~m}, 10 \mathrm{H}, 10$ $\mathrm{ArH})$.
${ }^{13} \mathrm{C}$ NMR $\left(100 \mathrm{MHz}, \mathrm{CDCl}_{3}\right): \delta=39.8\left(\mathrm{CH}_{2} \mathrm{CH}_{2} \mathrm{OH}\right), 47.5$ $\left(\mathrm{CH}_{2} \mathrm{COH}\right), 60.7\left(\mathrm{CH}_{2} \mathrm{OH}\right), 76.8(\mathrm{COH}), 117.8\left(\mathrm{H}_{2} \mathrm{C}=\mathrm{C}\right), 125.9$, 126.8, 128.0 (10 ArCH), 142.4 (2 $\mathrm{ArC}), 146.8\left(C=\mathrm{CH}_{2}\right)$.

MS (EI): $m / z(\%)=250\left[\mathrm{M}^{+}-\mathrm{H}_{2} \mathrm{O}\right.$, ] (29), 232 (10), 220 (18), 217 (16), 206 (14), 205 (54), 204 (22), 203 (23), 202 (20), 184 (12), 183 (84), 182 (24), 178 (11), 165 (13), 128 (10), 105 (100), 91 (10), 77 (49), 51 (10).

HRMS: $m / z,\left[\mathrm{M}^{+}\right]$calcd for $\mathrm{C}_{18} \mathrm{H}_{20} \mathrm{O}_{2}: 268.1463 ; m / z,\left[\mathrm{M}^{+}-\mathrm{H}_{2} \mathrm{O}\right]$ calcd for $\mathrm{C}_{18} \mathrm{H}_{18} \mathrm{O}$ : 250.1358; found: 250.1348 .

\section{3-Methylenenonane-1,5-diol (9d)}

Colorless oil; GLC: $t_{\mathrm{R}}=10.65 \mathrm{~min} ; R_{f}=0.33$ (hexane-EtOAc, $1: 1)$.

IR (film): $3345(\mathrm{OH}), 3075,1644 \mathrm{~cm}^{-1}(\mathrm{CH}=\mathrm{C})$.

${ }^{1} \mathrm{H}$ NMR (400 MHz, $\mathrm{CDCl}_{3}$ ): $\delta=0.92\left(\mathrm{t}, J=7.0 \mathrm{~Hz}, 3 \mathrm{H}, \mathrm{CH}_{3}\right.$ ), $1.20-1.55\left[\mathrm{~m}, 6 \mathrm{H},\left(\mathrm{CH}_{2}\right)_{3}\right], 1.96(\mathrm{br} \mathrm{s}, 2 \mathrm{H}, 2 \mathrm{OH}), 2.10$ (dd, $J=9.8$, $\left.6.9 \mathrm{~Hz}, 1 \mathrm{H}, \mathrm{CH}_{\mathrm{A}} \mathrm{H}_{\mathrm{B}} \mathrm{CHOH}\right), 2.25-2.40\left(\mathrm{~m}, 3 \mathrm{H}, \mathrm{CH}_{2} \mathrm{CH}_{2} \mathrm{OH}\right.$, $\left.\mathrm{CH}_{\mathrm{A}} \mathrm{H}_{\mathrm{B}} \mathrm{CHOH}\right), 3.65-3.85\left(\mathrm{~m}, 3 \mathrm{H}, \mathrm{CHOH}, \mathrm{CH}_{2} \mathrm{OH}\right), 4.99(\mathrm{~s}, 2 \mathrm{H}$, $\mathrm{H}_{2} \mathrm{C}=\mathrm{C}$ ).

${ }^{13} \mathrm{C}$ NMR $\left(100 \mathrm{MHz}, \mathrm{CDCl}_{3}\right): \delta=14.1 \quad\left(\mathrm{CH}_{3}\right), 22.7,27.9$ $\left[\mathrm{CH}_{2} \mathrm{CH}_{2} \mathrm{CH}_{3}\right], 37.0\left[\mathrm{CH}_{2} \mathrm{CH}_{2} \mathrm{CHOH}\right], 38.9\left(\mathrm{CH}_{2} \mathrm{CH}_{2} \mathrm{OH}\right), 44.1$ $\left(\mathrm{CCH}_{2} \mathrm{CHOH}\right), 60.64\left(\mathrm{CH}_{2} \mathrm{OH}\right), 69.6(\mathrm{CHOH}), 114.8\left(\mathrm{H}_{2} \mathrm{C}=\mathrm{C}\right)$, $143.6\left(C=\mathrm{CH}_{2}\right)$.

MS (EI): $m / z(\%)=172\left[\mathrm{M}^{+}\right](<1), 154\left[\mathrm{M}^{+}-\mathrm{H}_{2} \mathrm{O}\right](2), 117(25)$, 87 (65), 85 (12), 69 (100), 68 (68), 67 (50), 57 (19), 56 (32), 55 (11), 53 (11).

HRMS: $m / z\left[\mathrm{M}^{+}\right]$calcd for $\mathrm{C}_{10} \mathrm{H}_{20} \mathrm{O}_{2}: 172.1463 ; \mathrm{m} / z,\left[\mathrm{M}^{+}-\mathrm{H}_{2} \mathrm{O}\right]$ calcd for $\mathrm{C}_{10} \mathrm{H}_{18} \mathrm{O}$ : 154.1358 ; found: 154.1351 .

\section{1-Cyclohexyl-3-methylenepentane-1,5-diol (9e)}

Colorless oil; GLC: $t_{\mathrm{R}}=12.96 \mathrm{~min} ; R_{f}=0.33$ (hexane-EtOAc, $1: 1)$.

IR (KBr): $3354(\mathrm{OH}), 3075,1644 \mathrm{~cm}^{-1}\left(\mathrm{CH}_{2}=\mathrm{C}\right)$.

${ }^{1} \mathrm{H}$ NMR (300 MHz, $\mathrm{CDCl}_{3}$ ): $\delta=0.85-1.48,1.62-1.91[2 \mathrm{~m}, 11 \mathrm{H}$, $\left.\left(\mathrm{CH}_{2}\right)_{5} \mathrm{CH}\right], 1.92-2.15,2.26-2.40\left(2 \mathrm{~m}, 6 \mathrm{H}, 2 \mathrm{OH}, \mathrm{CH}_{2} \mathrm{CCH}_{2}\right), 3.51$ (ddd, $J=10.3,5.6,2.9 \mathrm{~Hz}, 1 \mathrm{H}, \mathrm{CHOH}), 3.75(\mathrm{t}, J=6.2 \mathrm{~Hz}, 2 \mathrm{H}$, $\left.\mathrm{CH}_{2} \mathrm{OH}\right), 5.00\left(\mathrm{~s}, 2 \mathrm{H}, \mathrm{H}_{2} \mathrm{C}=\mathrm{C}\right)$.

${ }^{13} \mathrm{C}$ NMR (75 MHz, $\left.\mathrm{CDCl}_{3}\right): \delta=26.1,26.2,26.5,28.2,29.0$ $\left[\left(\mathrm{CH}_{2}\right)_{5}\right], 38.9\left(\mathrm{CH}_{2} \mathrm{CH}_{2} \mathrm{OH}\right), 40.8\left(\mathrm{CH}_{2} \mathrm{CHOH}\right), 43.6(\mathrm{CHCOH})$, $60.6\left(\mathrm{CH}_{2} \mathrm{OH}\right), 73.5(\mathrm{CHOH}), 114.7\left(\mathrm{H}_{2} \mathrm{C}=\mathrm{C}\right), 144.0\left(C=\mathrm{CH}_{2}\right)$.

MS (EI): $m / z(\%)=180\left[\mathrm{M}^{+}-\mathrm{H}_{2} \mathrm{O}\right](<1 \%), 143(11), 113(44), 95$ (100), 83 (26), 69 (15), 68 (27), 67 (28), 56 (11), 55 (30).

HRMS: $m / z\left[\mathrm{M}^{+}\right]$calcd for $\mathrm{C}_{12} \mathrm{H}_{22} \mathrm{O}_{2}: 198.1620 ; \mathrm{m} / z\left[\mathrm{M}^{+}-\mathrm{H}_{2} \mathrm{O}\right]$ calcd for $\mathrm{C}_{12} \mathrm{H}_{20} \mathrm{O}$ : 180.1514; found: 180.1531 .

\section{3-Methylene-1-phenylpentane-1,5-diol (9f)}

Colorless oil; GLC: $t_{\mathrm{R}}=13.07 \mathrm{~min} ; R_{f}=0.33$ (hexane-EtOAc, $1: 1)$.

IR (KBr): 3354 (OH), 3064, 3029, 1644, $1494 \mathrm{~cm}^{-1}(\mathrm{CH}=\mathrm{C})$.

${ }^{1} \mathrm{H}$ NMR $\left(300 \mathrm{MHz}, \mathrm{CDCl}_{3}\right): \delta=2.25-2.57\left(\mathrm{~m}, 4 \mathrm{H}, \mathrm{CH}_{2} \mathrm{CCH}_{2}\right)$, $3.78\left(\mathrm{t}, J=5.9 \mathrm{~Hz}, 2 \mathrm{H}, \mathrm{CH}_{2} \mathrm{OH}\right), 4.75-4.90(\mathrm{~m}, 1 \mathrm{H}, \mathrm{CHOH}), 5.04$, $5.05\left(2 \mathrm{~s}, 2 \mathrm{H}, \mathrm{H}_{2} \mathrm{C}=\mathrm{C}\right), 7.20-7.45(\mathrm{~m}, 5 \mathrm{H}, 5 \mathrm{ArH})$.

${ }^{13} \mathrm{C}$ NMR $\left(75 \mathrm{MHz}, \mathrm{CDCl}_{3}\right): \delta=39.0 \quad\left(\mathrm{CH}_{2} \mathrm{CH}_{2} \mathrm{OH}\right), 46.1$ $\left(\mathrm{CH}_{2} \mathrm{CHOH}\right), 60.6\left(\mathrm{CH}_{2} \mathrm{OH}\right), 72.5(\mathrm{CHOH}), 115.4\left(\mathrm{H}_{2} \mathrm{C}=\mathrm{C}\right), 125.7$, 127.6, 128.5 (5 ArH), 143.1 ( $\mathrm{ArC}), 144.0\left(C=\mathrm{CH}_{2}\right)$.

MS (EI): $m / z(\%)=192\left[\mathrm{M}^{+}\right](<1), 174\left[\mathrm{M}^{+}-\mathrm{H}_{2} \mathrm{O}\right](4), 129(10)$, 107 (100), 105 (14), 79 (45), 77 (30).

HRMS: $m / z\left[\mathrm{M}^{+}\right]$calcd for $\mathrm{C}_{12} \mathrm{H}_{16} \mathrm{O}_{2}: 192.1150 ; m / z,\left[\mathrm{M}^{+}-\mathrm{H}_{2} \mathrm{O}\right]$ calcd for $\mathrm{C}_{12} \mathrm{H}_{14} \mathrm{O}$ : 174.1045; found: 174.101 . 
Palladium-Catalyzed Cyclization of the Methylene-1,5-diols 9; General Procedure

A soln of $\mathrm{PdCl}_{2}(8.9 \mathrm{mg}, 0.05 \mathrm{mmol}), \mathrm{CuCl}_{2}(67.2 \mathrm{mg}), \mathrm{MeOH}(10$ $\mathrm{mL})$, and the corresponding methylene-1,5-diol 9 (1 mmol) was prepared in a screw-top tube, followed by the addition of $35 \% \mathrm{H}_{2} \mathrm{O}_{2}$ soln $(0.86 \mathrm{~mL}, 10 \mathrm{mmol})$. The top was airtight on the reaction tube, which was heated at $70{ }^{\circ} \mathrm{C}$ for $24 \mathrm{~h}$. The solvent was evaporated to dryness, followed by the addition of EtOAc $(20 \mathrm{~mL})$ and filtration through Celite. The filtrate was washed with brine $(2 \times 5 \mathrm{~mL})$, the organic phase was dried (anhyd $\mathrm{MgSO}_{4}$ ), and the solvent evaporated under vacuum (20 mbar). Compounds 10a and 10b did not require any further purification, while compounds $10 \mathbf{c}-\mathbf{f}$ were purified by column chromatography (silica gel, hexane-EtOAc,).

\section{$\left(3 \mathrm{a} R *, 6 \mathrm{a} S^{*}\right)$-2,2-Diethylhexahydrofuro[2,3-b]furan (10a)}

Colorless oil; GLC: $t_{\mathrm{R}}=9.28 \mathrm{~min} ; R_{f}=0.48$ (hexane-EtOAc, 8:2). IR (film): $1024 \mathrm{~cm}^{-1}(\mathrm{C}-\mathrm{O})$.

${ }^{1} \mathrm{H}$ NMR $\left(400 \mathrm{MHz}, \mathrm{CDCl}_{3}\right): \delta=0.85,0.90(2 \mathrm{t}, J=7.5 \mathrm{~Hz}, 6 \mathrm{H}, 2$ $\left.\mathrm{CH}_{3}\right), 1.35-1.75,1.88-2.07\left(2 \mathrm{~m}, 8 \mathrm{H}, 2 \mathrm{CH}_{2} \mathrm{CH}_{3}, \mathrm{CH}_{2} \mathrm{CHCH}_{2}\right.$ ), 2.85-3.00 (m, $\left.1 \mathrm{H}, \mathrm{CH}_{2} \mathrm{CHCH}_{2}\right), 3.84-3.95\left(\mathrm{~m}, 2 \mathrm{H}, \mathrm{CH}_{2} \mathrm{O}\right), 5.68$ $(\mathrm{d}, J=5.1 \mathrm{~Hz}, 1 \mathrm{H}, \mathrm{OCHO})$.

${ }^{13} \mathrm{C}$ NMR $\left(100 \mathrm{MHz}, \mathrm{CDCl}_{3}\right): \delta=8.4,8.7\left(2 \mathrm{CH}_{3}\right), 30.5,31.2(2$ $\left.\mathrm{CH}_{2} \mathrm{CH}_{3}\right), \quad 32.8 \quad\left(\mathrm{CH}_{2} \mathrm{CH}_{2} \mathrm{CO}\right), \quad 39.2 \quad\left(\mathrm{CHCH}_{2} \mathrm{COC}\right), \quad 43.1$ $\left(\mathrm{CH}_{2} \mathrm{CHCH}_{2}\right), 65.8\left(\mathrm{CH}_{2} \mathrm{O}\right), 88.0(\mathrm{CO}), 109.1(\mathrm{OCHO})$.

MS (EI): $m / z(\%)=170\left[\mathrm{M}^{+}\right](<1), 141(100), 95$ (20), 57 (55), 55 (15).

HRMS: $\mathrm{m} / z\left[\mathrm{M}^{+}\right]$calcd for $\mathrm{C}_{10} \mathrm{H}_{18} \mathrm{O}_{2}: 170.1307 ; \mathrm{m} / z\left(\mathrm{M}^{+}-\mathrm{C}_{2} \mathrm{H}_{5}\right)$ calcd for $\mathrm{C}_{8} \mathrm{H}_{13} \mathrm{O}_{2}$ : 141.0910; found: 141.0899 .

$\left(3 a^{\prime} R^{*}, 6 a^{\prime} S^{*}\right)$-Tetrahydro-3'H-spiro[cyclohexane-1,2'-furo[2,3b] furan] (10b)

Colorless oil; GLC: $t_{\mathrm{R}}=11.02 \mathrm{~min} ; R_{f}=0.40$ (hexane-EtOAc, $8: 2)$.

IR (KBr): $1020 \mathrm{~cm}^{-1}(\mathrm{C}-\mathrm{O})$.

${ }^{1} \mathrm{H}$ NMR $\left(300 \mathrm{MHz}, \mathrm{CDCl}_{3}\right): \delta=1.20-1.80,1.85-2.14(2 \mathrm{~m}, 14 \mathrm{H}$, $\left.7 \mathrm{CH}_{2}\right), 2.85-3.01\left(\mathrm{~m}, 1 \mathrm{H}, \mathrm{CH}_{2} \mathrm{CHCH}_{2}\right), 3.80-4.00(\mathrm{~m}, 2 \mathrm{H}$, $\left.\mathrm{CH}_{2} \mathrm{O}\right), 5.68(\mathrm{~d}, J=5.2 \mathrm{~Hz}, 1 \mathrm{H}, \mathrm{OCHO})$.

${ }^{13} \mathrm{C}$ NMR $\left(75 \mathrm{MHz}, \mathrm{CDCl}_{3}\right): \delta=23.3,23.7,25.4\left[2 \mathrm{CH}_{2} \mathrm{CH}_{2} \mathrm{C}\right.$, $\left.\mathrm{CH}_{2}\left(\mathrm{CH}_{2}\right)_{2} \mathrm{C}\right], 32.5\left(\mathrm{CH}_{2} \mathrm{CH}_{2} \mathrm{O}\right), 36.9,38.1\left(2 \mathrm{CH}_{2} \mathrm{CH}_{2} \mathrm{C}\right), 41.2$ $\left(\mathrm{CCH}_{2} \mathrm{CH}\right), 42.4\left(\mathrm{CH}_{2} \mathrm{CHCH}_{2}\right), 65.6\left(\mathrm{CH}_{2} \mathrm{O}\right), 84.3(\mathrm{CO}), 108.4$ (OCHO).

MS (EI): $m / z(\%)=182\left[\mathrm{M}^{+}\right](20), 140(19), 139(100), 126(30)$, 121 (11), 84 (10), 82 (25), 81 (13), 67 (11), 55 (23).

HRMS: $m / z\left[\mathrm{M}^{+}\right]$calcd for $\mathrm{C}_{11} \mathrm{H}_{18} \mathrm{O}_{2}$ : 182.1307; found: 182.1332 .

$(3 \mathrm{a} R *, 6 \mathrm{a} S *)$-2,2-Diphenylhexahydrofuro[2,3-b]furan (10c) White solid; mp $95{ }^{\circ} \mathrm{C}$; GLC: $t_{\mathrm{R}}=16.46 \mathrm{~min} ; R_{f}=0.46$ (hexaneEtOAc, 8:2).

IR (KBr): 3053, 3021, 1595, $1490(\mathrm{CH}=\mathrm{C}), 1011 \mathrm{~cm}^{-1}(\mathrm{C}-\mathrm{O})$.

${ }^{1} \mathrm{H}$ NMR (300 MHz, $\mathrm{CDCl}_{3}$ ): $\delta=1.65(\mathrm{dd}, J=6.2,5.0 \mathrm{~Hz}, 1 \mathrm{H}$, $\left.\mathrm{CH}_{\mathrm{A}} \mathrm{H}_{\mathrm{B}} \mathrm{CH}_{2} \mathrm{O}\right), 1.84-1.98\left(\mathrm{~m}, 1 \mathrm{H}, \mathrm{CH}_{\mathrm{A}} H_{\mathrm{B}} \mathrm{CH}_{2} \mathrm{O}\right), 2.15$ (dd, $J=8.5$, $\left.6.3 \mathrm{~Hz}, 1 \mathrm{H}, \mathrm{CH}_{\mathrm{A}} \mathrm{H}_{\mathrm{B}} \mathrm{C}\right), 2.75-2.90\left(\mathrm{~m}, 1 \mathrm{H}, \mathrm{CH}_{2} \mathrm{CHCH}_{2}\right), 3.03(\mathrm{dd}$, $\left.J=8.7,6.3 \mathrm{~Hz}, 1 \mathrm{H}, \mathrm{CH}_{\mathrm{A}} H_{\mathrm{B}} \mathrm{C}\right), 3.75-3.85\left(\mathrm{~m}, 1 \mathrm{H}, \mathrm{CH}_{\mathrm{A}} \mathrm{H}_{\mathrm{B}} \mathrm{O}\right), 3.93$ $\left(\mathrm{dd}, J=6.8,5.7 \mathrm{~Hz}, 1 \mathrm{H}, \mathrm{CH}_{\mathrm{A}} H_{\mathrm{B}} \mathrm{O}\right), 5.79(\mathrm{~d}, J=5.3 \mathrm{~Hz}, 1 \mathrm{H}$, OCHO), 7.10-7.60 (m, $10 \mathrm{H}, 10 \mathrm{ArH})$.

${ }^{13} \mathrm{C}$ NMR $\left(75 \mathrm{MHz}, \mathrm{CDCl}_{3}\right): \delta=31.9\left(\mathrm{CH}_{2} \mathrm{CH}_{2} \mathrm{O}\right), 42.7\left(\mathrm{CH}_{2} \mathrm{C}\right)$, $43.1\left(\mathrm{CH}_{2} \mathrm{CHCH}_{2}\right), 66.5\left(\mathrm{CH}_{2} \mathrm{O}\right), 88.6(\mathrm{CO}), 108.7$ (OCHO), 125.4, 125.6, 126.8, 128.0, 128.2 (10 ArCH), 145.3, 145.9 (2 ArC).

MS (EI): $m / z(\%)=266\left[\mathrm{M}^{+}\right](10), 190(10), 189(62), 184(14), 183$ (100), 178 (12), 165 (18), 115 (10), 105 (48), 91 (12), 84 (13), 77 (19).

HRMS: $m / z\left[\mathrm{M}^{+}\right]$calcd for $\mathrm{C}_{18} \mathrm{H}_{18} \mathrm{O}_{2}$ : 266.1307; found: 266.1271 . $\left(2 R^{*}, 3 \mathrm{a} R^{*}, 6 \mathrm{a} S^{*}\right)-2-$-Butylhexahydrofuro[2,3-b]furan (10d)

Colorless oil; GLC: $t_{\mathrm{R}}=10.70 \mathrm{~min} ; R_{f}=0.50$ (hexane-EtOAc, 8:2). IR (KBr): $1015 \mathrm{~cm}^{-1}(\mathrm{C}-\mathrm{O})$.

${ }^{1} \mathrm{H}$ NMR $\left(300 \mathrm{MHz}, \mathrm{CDCl}_{3}\right): \delta=0.90\left(\mathrm{t}, J=6.9 \mathrm{~Hz}, 3 \mathrm{H}, \mathrm{CH}_{3}\right)$, 1.20-1.50 [m, $\left.6 \mathrm{H},\left(\mathrm{CH}_{2}\right)_{3}\right], 1.50-1.78,1.78-1.90,2.04-2.20(3 \mathrm{~m}$, $\left.4 \mathrm{H}, \mathrm{CH}_{2} \mathrm{CHCH}_{2}\right), 2.75-2.95\left(\mathrm{~m}, 1 \mathrm{H}, \mathrm{CH}_{2} \mathrm{CHCH}_{2}\right), 3.79-3.99$ (m, $\left.2 \mathrm{H}, \mathrm{CH}_{2} \mathrm{O}\right), 4.00-4.15(\mathrm{~m}, 1 \mathrm{H}, \mathrm{CHO}), 5.71(\mathrm{~d}, J=5.1 \mathrm{~Hz}, 1 \mathrm{H}$, $\mathrm{OCHO}$ ).

${ }^{13} \mathrm{C} \quad \mathrm{NMR} \quad\left(75 \mathrm{MHz}, \mathrm{CDCl}_{3}\right): \delta=14.0 \quad\left(\mathrm{CH}_{3}\right), \quad 22.7,28.3$ $\left(\mathrm{CH}_{3} \mathrm{CH}_{2} \mathrm{CH}_{2}\right), 32.7,35.3,38.8\left(\mathrm{CH}_{2} \mathrm{CHCH}_{2}, \mathrm{CH}_{3} \mathrm{CH}_{2} \mathrm{CH}_{2} \mathrm{CH}_{2}\right)$, $42.6\left(\mathrm{CH}_{2} \mathrm{CHCH}_{2}\right), 68.2\left(\mathrm{CH}_{2} \mathrm{O}\right), 79.7(\mathrm{CHO}), 108.9$ (OCHO).

MS (EI): $\mathrm{m} / z(\%)=170\left[\mathrm{M}^{+}\right](<1), 113(100), 84$ (12), 69 (48), 67 (10), 55 (16).

HRMS: $m / z\left[\mathrm{M}^{+}\right]$calcd for $\mathrm{C}_{10} \mathrm{H}_{18} \mathrm{O}_{2}: 170.1307$; found: 170.1332 .

Selected data for the minor diastereomer $\left(2 S^{*}, 3 \mathrm{a} R^{*}, 6 \mathrm{a} S^{*}\right)-\mathbf{1 0 d}$ :

GLC: $t_{\mathrm{R}}=10.60 \mathrm{~min}$.

${ }^{1} \mathrm{H}$ NMR (400 MHz, $\left.\mathrm{CDCl}_{3}\right): \delta=5.63(\mathrm{~d}, J=5.3 \mathrm{~Hz}, 1 \mathrm{H}, \mathrm{OCHO})$. MS (EI): $\mathrm{m} / z(\%)=170\left[\mathrm{M}^{+}\right](<1), 113(100), 84$ (15), 69 (47), 67 (11), 55 (17).

\section{$\left(2 R^{*}, 3 \mathrm{a} S^{*}, 6 \mathrm{a} R^{*}\right)-2-C y c l o h e x y l h e x a h y d r o f u r o[2,3-b]$ furan $(10 \mathrm{e})^{8 \mathrm{~b}}$}

Colorless oil; GLC: $t_{\mathrm{R}}=12.57 \mathrm{~min} ; R_{f}=0.55$ (hexane-EtOAc, 8:2). IR (KBr): 1018, $1097 \mathrm{~cm}^{-1}(\mathrm{C}-\mathrm{O})$.

${ }^{1} \mathrm{H}$ NMR $\left(400 \mathrm{MHz}, \mathrm{CDCl}_{3}\right): \delta=0.85-1.10,1.10-1.47,1.50-1.85$, 1.90-2.20 [4 m, $\left.14 \mathrm{H},\left(\mathrm{CH}_{2}\right)_{5}, \mathrm{CH}_{2} \mathrm{CHCH}_{2}\right], 2.75-2.95(\mathrm{~m}, 1 \mathrm{H}$, $\mathrm{CH}_{2} \mathrm{CHCH}_{2}$ ), 3.61-3.97 (m, $\left.3 \mathrm{H}, \mathrm{CH}_{2} \mathrm{O}, \mathrm{CHO}\right), 5.70(\mathrm{~d}, J=5.0 \mathrm{~Hz}$, $1 \mathrm{H}, \mathrm{OCHO})$.

${ }^{13} \mathrm{C}$ NMR $\left(100 \mathrm{MHz}, \mathrm{CDCl}_{3}\right): \delta=25.8,26.0,26.4,28.8,30.0$ $\left[\left(\mathrm{CH}_{2}\right)_{5}\right], 32.8\left(\mathrm{CH}_{2} \mathrm{CH}_{2} \mathrm{O}\right), 36.5\left(\mathrm{CH}_{2} \mathrm{CHCH}_{2}\right), 68.2\left(\mathrm{CH}_{2} \mathrm{O}\right), 84.2$ (CHO), 108.7 (OCHO).

MS (EI): $m / z(\%)=196\left[\mathrm{M}^{+}\right](<1), 152(10), 113(100), 69(37), 55$ (13).

HRMS: $m / z\left[\mathrm{M}^{+}\right]$calcd for $\mathrm{C}_{12} \mathrm{H}_{20} \mathrm{O}_{2}: 196.1463$; found: 196.1432 .

Selected data for the minor diastereomer $\left(2 S^{*}, 3 \mathrm{a} S^{*}, 6 \mathrm{a} R^{*}\right)-\mathbf{1 0 e}$ :

GLC: $t_{\mathrm{R}}=12.45 \mathrm{~min}$.

${ }^{1} \mathrm{H}$ NMR (400 MHz, $\left.\mathrm{CDCl}_{3}\right): \delta=5.62(\mathrm{~d}, J=5.5 \mathrm{~Hz}, 1 \mathrm{H}, \mathrm{OCHO})$. MS (EI): $m / z(\%)=196\left[\mathrm{M}^{+}\right](<1), 113$ (100), 69 (37), 55 (13).

$\left(2 R^{*}, 3 \mathrm{a} S^{*}, 6 \mathrm{a} R^{*}\right)$-2-Phenylhexahydrofuro[2,3-b]furan $(10 \mathrm{f})^{8 \mathrm{~b}}$ Colorless oil; GLC: $t_{\mathrm{R}}=12.91 \mathrm{~min} ; R_{f}=0.35$ (hexane-EtOAc, $8: 2)$.

IR (KBr): 3062, 3030, 1603, $1494(\mathrm{CH}=\mathrm{C}), 1016 \mathrm{~cm}^{-1}(\mathrm{C}-\mathrm{O})$.

${ }^{1} \mathrm{H}$ NMR $\left(400 \mathrm{MHz}, \mathrm{CDCl}_{3}\right): \delta=1.80-1.90,1.98-2.10,2.14-2.30$ ( $3 \mathrm{~m}, 4 \mathrm{H}, \mathrm{CH}_{2} \mathrm{CHCH}_{2}$ ), 2.97-3.09 (m, $1 \mathrm{H}, \mathrm{CH}_{2} \mathrm{CHCH}_{2}$ ), 3.91$4.10\left(\mathrm{~m}, 2 \mathrm{H}, \mathrm{CH}_{2} \mathrm{O}\right), 5.12(\mathrm{dd}, J=5.7,5.0 \mathrm{~Hz}, 1 \mathrm{H}, \mathrm{CHO}), 5.93(\mathrm{~d}$, $J=4.9 \mathrm{~Hz}, 1 \mathrm{H}, \mathrm{OCHO}), 7.24-7.46$ (m, $5 \mathrm{H}, 5 \mathrm{ArH})$.

${ }^{13} \mathrm{C}$ NMR $\left(100 \mathrm{MHz}, \mathrm{CDCl}_{3}\right): \delta=32.5 \quad\left(\mathrm{CH}_{2} \mathrm{CH}_{2} \mathrm{O}\right), 41.6$ $\left(\mathrm{CH}_{2} \mathrm{CHO}\right), 43.0\left(\mathrm{CH}_{2} \mathrm{CHCH}_{2}\right), 68.4\left(\mathrm{CH}_{2} \mathrm{O}\right), 84.8(\mathrm{CHO}), 109.4$ (OCHO), 125.7, 127.5, 128.4 (5 ArCH), 141.5 (ArC).

MS (EI): $m / z(\%)=190\left[\mathrm{M}^{+}\right](10 \%, 145(10), 143(10), 129$ (38), 128 (15), 117 (13), 115 (17), 107 (20), 105 (21), 104 (21), 91 (22), 84 (100), 83 (19), 78 (10), 77 (22), 70 (27), 69 (14), 56 (15), 55 (20). HRMS: $\mathrm{m} / z\left[\mathrm{M}^{+}\right]$calcd for $\mathrm{C}_{12} \mathrm{H}_{14} \mathrm{O}_{2}$ : 190.0994; found: 190.0989 . Selected data for the minor diastereomer $\left(2 S^{*}, 3 \mathrm{a} S^{*}, 6 \mathrm{a} R^{*}\right)-\mathbf{1 0 f}$ : GLC: $t_{\mathrm{R}}=12.45 \mathrm{~min}$.

${ }^{1} \mathrm{H}$ NMR (400 MHz, $\left.\mathrm{CDCl}_{3}\right): \delta=5.81(\mathrm{~d}, J=5.5 \mathrm{~Hz}, 1 \mathrm{H}, \mathrm{OCHO})$. 
MS (EI): $m / z(\%)=190\left[\mathrm{M}^{+}\right](6), 129(31), 128(16), 117(11), 115$ (16), 107 (21), 105 (21), 104 (22), 91 (21), 84 (100), 83 (19), 77 (22), 70 (22), 69 (14), 56 (15), 55 (20).

\section{$\left(3 \mathrm{a} R *, 6 \mathrm{a} S^{*}\right)-5,5-D i e t h y l t e t r a h y d r o f u r o[2,3-b]$ furan-2(6a $\left.H\right)$ -} one

Following a variant of a literature procedure for the oxidation of a tetrahydrofuran ring: ${ }^{23}$ Compound $\mathbf{1 0 a}(170 \mathrm{mg}, 1 \mathrm{mmol})$ was slowly added to a soln of $\mathrm{RuO}_{2} \cdot \mathrm{x} \mathrm{H}_{2} \mathrm{O}(27 \mathrm{mg})$ and $\mathrm{NaIO}_{4}(856 \mathrm{mg}, 4$ mmol) in $\mathrm{CH}_{2} \mathrm{Cl}_{2}-\mathrm{H}_{2} \mathrm{O}-\mathrm{MeCN}(2: 2: 1,5 \mathrm{~mL})$. The mixture was stirred at r.t. for $6 \mathrm{~h}$, followed by the addition of $\mathrm{H}_{2} \mathrm{O}(10 \mathrm{~mL})$ and extraction with $\mathrm{CH}_{2} \mathrm{Cl}_{2}(3 \times 10 \mathrm{~mL})$. The combined organic extracts were dried (anhyd $\mathrm{MgSO}_{4}$ ), concentrated under vacuum (20 mbar), and purified by column chromatography (silica gel, hexane-EtOAc, 1:1) to give pure lactone 11a as a colorless oil; GLC: $t_{\mathrm{R}}=11.52$ $\min ; R_{f}=0.51$ (hexane-EtOAc, 1:1).

IR (film): $1778(\mathrm{C}=\mathrm{O}), 1116 \mathrm{~cm}^{-1}(\mathrm{C}-\mathrm{O})$.

${ }^{1} \mathrm{H} \mathrm{NMR}\left(400 \mathrm{MHz}, \mathrm{CDCl}_{3}\right): \delta=0.89\left(2 \mathrm{t}, 6 \mathrm{H}, J=7.5, \mathrm{~Hz}, 2 \mathrm{CH}_{3}\right)$, 1.50-1.75 (m, $\left.5 \mathrm{H}, 2 \mathrm{CH}_{2} \mathrm{CH}_{3}, \mathrm{CH}_{\mathrm{A}} \mathrm{H}_{\mathrm{B}} \mathrm{CO}\right), 2.19$ (dd, $J=13.2,9.9$ $\left.\mathrm{Hz}, 1 \mathrm{H}, \mathrm{CH}_{\mathrm{A}} H_{\mathrm{B}} \mathrm{CO}\right), 2.49\left(\mathrm{dd}, J=17.9,1.3 \mathrm{~Hz}, 1 \mathrm{H}, \mathrm{CH}_{\mathrm{A}} \mathrm{H}_{\mathrm{B}} \mathrm{CO}_{2}\right)$, $2.79\left(\mathrm{dd}, J=17.9,8.5 \mathrm{~Hz}, 1 \mathrm{H}, \mathrm{CH}_{\mathrm{A}} H_{\mathrm{B}} \mathrm{CO}_{2}\right), 3.07-3.18(\mathrm{~m}, 1 \mathrm{H}$, $\left.\mathrm{CH}_{2} \mathrm{CHCH}_{2}\right), 6.01(\mathrm{~d}, J=5.1 \mathrm{~Hz}, 1 \mathrm{H}, \mathrm{OCHO})$.

${ }^{13} \mathrm{C} \mathrm{NMR}\left(100 \mathrm{MHz}, \mathrm{CDCl}_{3}\right): \delta=8.3,8.5\left(2 \mathrm{CH}_{3}\right), 31.1,32.2(2$ $\left.\mathrm{CH}_{2} \mathrm{CH}_{3}\right), 36.5\left(\mathrm{CH}_{2} \mathrm{CO}_{2}\right), 39.5\left(\mathrm{CH}_{2} \mathrm{CO}\right), 40.1\left(\mathrm{CH}_{2} \mathrm{CHCH}_{2}\right), 92.2$ (CO), 109.1 (OCHO), $174.4\left(\mathrm{CO}_{2}\right)$.

MS (EI): $m / z(\%)=184\left[\mathrm{M}^{+}\right](<1), 155\left[\mathrm{M}^{+}-\mathrm{C}_{2} \mathrm{H}_{5}\right](100), 137(18)$, 127 (14), 112 (10), 111 (11), 109 (13), 97 (10), 96 (15), 81 (12), 70 (10), 69 (17), 57 (41), 55 (16).

HRMS: $m / z,\left[\mathrm{M}^{+}\right]$calcd for $\mathrm{C}_{10} \mathrm{H}_{16} \mathrm{O}_{3}: 184.1099 ; \mathrm{m} / z\left[\mathrm{M}^{+}-\mathrm{C}_{2} \mathrm{H}_{5}\right]$ calcd $\mathrm{C}_{8} \mathrm{H}_{11} \mathrm{O}_{3}$ : 155.0703; found: 155.0737 .

\section{Acknowledgment}

This work was generously supported by the Spanish Ministerio de Educación y Ciencia (MEC; grant no. CTQ2007-65218 and Consolider Ingenio 2010-CSD2007-00006) and the Generalitat Valenciana (grant no. PROMETEO/2009/039). D. S. and M. R.-F. thank the Vicerrectorado de Investigación, Desarrollo e Innovación of the University of Alicante for predoctoral and postdoctoral grants, respectively.

\section{References}

(1) (a) Cardillo, G.; Contento, M.; Sandri, S. Tetrahedron Lett. 1974, 15, 2215. (b) Carlson, R. M. Tetrahedron Lett. 1978, $19,111$.

(2) (a) Trost, B. M.; Chan, D. M. T. J. Am. Chem. Soc. 1981, 103, 5972. (b) Trost, B. M.; Chan, D. M. T.; Nanninga, T. N. Org. Synth. Coll. Vol. VII; John Wiley \& Sons: London, 1990, 266.

(3) Bigot, A.; Breit, B. Synthesis 2008, 3692.

(4) See, for instance: (a) Chen, H.; Tan, R. X.; Liu, Z. L.; Zhang, Y. J. Nat. Prod. 1996, 59, 668. (b) Boneva, I. M.; Malakov, P. Y.; Papanov, G. Y. Phytochemistry 1998, 47, 303. (c) Malakov, P. Y.; Papanov, G. Y. Phytochemistry 1998, 49, 2443.

(5) See, for instance: (a) Malakov, P. Y.; Papanov, G. Y.; Boneva, I. M. Phytochemistry 1996, 41, 855.

(b) de la Torre, M. C.; Rodríguez, B.; Bruno, B.; Vassallo, N.; Bondì, M. L.; Piozzi, F.; Servettaz, O. J. Nat. Prod. 1997, 60, 1229. (c) Malakov, P. Y.; Papanov, G. Y.
Phytochemistry 1997, 46, 955. (d) Malakov, P. Y.; Papanov, G. Y.; Deltchev, V. B. Phytochemistry 1998, 49, 811.

(6) (a) Rodríguez, B.; de la Torre, M. C.; Jimeno, M.-L.; Bruno, M.; Vassallo, N.; Bondi, M. L.; Piozzi, F.; Servettaz, O. J. Nat. Prod. 1997, 60, 348. (b) Malakov, P. Y.; Papanov, G. Y. Phytochemistry 1998, 49, 2449. (c) Bruno, M.; Cruciata, M.; Bondi, M. L.; Piozzi, F.; de la Torre, M. C.; Rodríguez, B.; Servettaz, O. Phytochemistry 1998, 48, 687.

(7) Rosselli, S.; Maggio, A.; Piozzi, F.; Simmonds, M. S. J.; Bruno, M. J. Agric. Food Chem. 2004, 52, 7867; and references cited therein.

(8) (a) Kojima, Y.; Kato, N. Tetrahedron 1981, 37, 2527. (b) klein Gebbinck, E. A.; Bouwman, C. T.; Bourgois, M.; Jansen, B. J. M.; de Groot, A. Tetrahedron 1999, 55, 11051

(9) Fukui, H.; Tsuchiya, Y.; Fujita, K.; Nakagawa, T.; Koshino, H.; Nakata, T. Bioorg. Med. Chem. Lett. 1997, 7, 2081.

(10) (a) Kido, F.; Sinha, S. C.; Abiko, T.; Watanabe, M.; Yoshikoshi, A. J. Chem. Soc., Chem. Commun. 1990, 418. (b) Kido, F.; Sinha, S. C.; Abiko, T.; Watanabe, M.; Yoshikoshi, A. Tetrahedron 1990, 46, 4887.

(11) (a) Alonso, F.; Lorenzo, E.; Yus, M. Tetrahedron Lett. 1997, 38, 2187. (b) Alonso, F.; Lorenzo, E.; Yus, M. Tetrahedron Lett. 1998, 39, 3303. (c) Lorenzo, E.; Alonso, F.; Yus, M. Tetrahedron Lett. 2000, 41, 1661. (d) Lorenzo, E.; Alonso, F.; Yus, M. Tetrahedron 2000, 56, 1745. (e) Alonso, F.; Lorenzo, E.; Meléndez, J.; Yus, M. Tetrahedron 2003, 59, 5199. (f) Alonso, F.; Meléndez, J.; Yus, M. Russ. Chem. Bull. 2003, 52, 2628. (g) Alonso, F.; Meléndez, J.; Yus, M. Tetrahedron Lett. 2005, 46, 6519. (h) Alonso, F.; Meléndez, J.; Yus, M. Tetrahedron 2006, 62, 4814.

(12) (a) Alonso, F.; Falvello, L. R.; Fanwick, P. E.; Lorenzo, E.; Yus, M. Synthesis 2000, 949. (b) Alonso, F.; Meléndez, J.; Yus, M. Helv. Chim. Acta 2002, 85, 3262. (c) Alonso, F.; Meléndez, J.; Yus, M. Tetrahedron Lett. 2004, 45, 1717. (d) Alonso, F.; Dacunha, B.; Meléndez, J.; Yus, M. Tetrahedron 2005, 61, 3437. (e) Dacunha, B.; Alonso, F.; Meléndez, J.; Yus, M. Acta Crystallogr., Sect. A 2005, 61, C157. (f) Meléndez, J.; Alonso, F.; Yus, M. Tetrahedron Lett. 2006, 47, 1187. (g) Alonso, F.; Meléndez, J.; Soler, T.; Yus, M. Tetrahedron 2006, 62, 2264. (h) Alonso, F.; Foubelo, F.; Yus, M. Curr. Chem. Biol. 2007, 1, 317. (i) Alonso, F.; Meléndez, J.; Yus, M. Synlett 2008, 1627.

(13) Alonso, F.; Sánchez, D.; Yus, M. Adv. Synth. Catal. 2008, $350,2118$.

(14) Moreno-Dorado, F.; Guerra, F. M.; Manzano, F. L.; Aladro, F. J.; Jorge, Z. D.; Massanet, G. M. Tetrahedron Lett. 2003, 44, 6691 .

(15) For a review, see: Yus, M. Synlett 2001, 1197.

(16) Yong, K. H.; Lotoski, J. A.; Chong, J. M. J. Org. Chem. 2001, 66, 8248.

(17) Cardillo, G.; Contento, M.; Sandri, S. J. Chem. Soc., Perkin Trans. 1 1979, 1729.

(18) For a review, see: Cravotto, G.; Cintas, P. Chem. Soc. Rev. 2006, 35, 180.

(19) Lecomte, V.; Stéphan, E.; Le Bideau, F.; Jaouen, G. Tetrahedron 2003, 59, 2169.

(20) Streiff, S.; Ribeiro, N.; Désaubry, L. Chem. Commun. 2004, 346.

(21) Stewart, J. J. P. J. Comput. Chem. 1991, 12, 320.

(22) For a review, see: Jung, M. E.; Pizzi, G. Chem. Rev. 2005, 105, 1735.

(23) Berkowitz, W. F.; Amarasekara, A. S.; Perumattam, J. J. J. Org. Chem. 1987, 52, 1119.

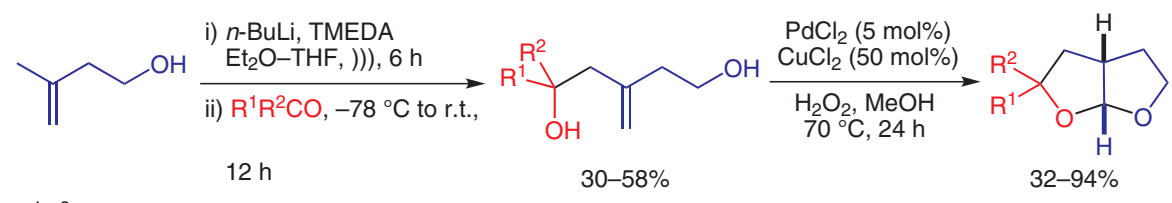

$\mathrm{R}^{1} \mathrm{R}^{2} \mathrm{CO}=\mathrm{Et}_{2} \mathrm{CO},\left(\mathrm{CH}_{2}\right)_{5} \mathrm{CO}, \mathrm{Ph}_{2} \mathrm{CO}, \mathrm{Me}\left(\mathrm{CH}_{2}\right)_{3} \mathrm{CHO}, c-\mathrm{C}_{6} \mathrm{H}_{11} \mathrm{CHO}, \mathrm{PhCHO}$ 